\title{
DETALLES TÉCNICOS Y MORBIMORTALIDAD DE LA FUNDOPLICATURA DE NISSEN LAPAROSCÓPICA EN 503 PACIENTES CON REFLUJO GASTROESOFÁGICO*
}

\author{
Drs. Attila Csendes ${ }^{1}$, Juan Carlos Díaz ${ }^{1}$, Patricio Burdiles ${ }^{1}$, Fernando Maluenda $^{1}$ \\ ${ }^{1}$ Departamento de Cirugía Hospital Clínico Universidad de Chile. \\ Santiago, Chile.
}

\begin{abstract}
\section{Laparoscopic Nissen fundoplication. Experience in 530 patients}

Background: Laparoscopic Nissen fundoplication is the surgical procedure of choice for patients with gastroesophageal reflux. Aim: To describe the most important surgical stages of the technique and report the rates of complications and mortality. Material and Methods: In a ten years period, 530 patients were subjected to laparoscopic fundoplication. In all patients a clinical history was obtained and an upper endoscopy with biopsy, esophageal manometry and $24 \mathrm{~h} \mathrm{pH}$ measurement were performed. Patients with Barret esophagus, hiatal hernia of more than $5 \mathrm{~cm}$ and those subjected to other surgical techniques were excluded from analysis. Results: No patient died. The conversion rate was $0.4 \%$. No patient required splenectomy. Two patients had complications and required a second intervention. Mean hospital stay was 2.8 days. Conclusions: Laparoscopic Nissen fundoplication is safe and has a low rate of complications.
\end{abstract}

Key words: Nissen fundoplication, Laparoscopic, Gastroesophageal reflux.

\section{Resumen}

Objetivo: El presente estudio pretende describir las etapas quirúrgicas más importantes de esta técnica y describir la morbimortalidad publicada por autores nacionales. Material y Método: Entre enero de 1993 y diciembre de 2013 un total de 530 pacientes se sometieron a una fundoplicatura laparoscópica dentro de un protocolo de estudio prospectivo. En todos se realizó una encuesta clínica, endoscopia con toma de biopsia, manometría esofágica y $\mathrm{pH}$ de $24 \mathrm{~h}$. Se excluyeron pacientes con esófago de Barrett, pacientes con hernia hiatal $>5 \mathrm{~cm}$ y pacientes sometidos a una técnica quirúrgica diferente. Resultados: La mortalidad operatoria fue cero. La tasa de conversión fue de $0,4 \%$. No hubo esplenectomía. Hubo un total de 2 pacientes complicados $(0,4 \%)$, siendo todos reoperados. La estadía hospitalaria fue de 2,8 días. Conclusión: La fundoplicatura de Nissen por vía laparoscópica es un procedimiento seguro, de muy baja morbilidad y nula mortalidad operatoria.

Palabras clave: Fundoplicatura, vasos cortos, hernia hiatal, reflujo gastroesofágico, Nissen.

\footnotetext{
*Recibido el 4 de mayo de 2015 y aceptado para publicación el 28 de septiembre de 2015.

Conflictos de interés: Ninguno
}

Correspondencia: Dr. Attila Csendes acsendes@hcuch.cl 


\section{Introducción}

La fundoplicatura de Nissen es la técnica quirúrgica más empleada para tratamiento de pacientes con reflujo gastroesofágico crónico patológico, tanto por vía laparotómica ${ }^{1,2}$ como por vía laparoscópica $^{3-6}$. Los resultados a largo plazo por más de 10 años han sido publicados previamente tanto por vía laparotómica ${ }^{7}$ como laparoscópica ${ }^{8}$. Sin embargo, en la literatura nacional persisten controversias sobre algunos puntos del procedimiento y muy poca referencia a la morbimortalidad de esta técnica por vía laparoscópica.

El objetivo principal del presente estudio fue analizar la morbilidad y mortalidad operatoria de nuestra experiencia comparada con los reportes de autores nacionales referentes a este tema. Como objetivos derivados se consideraron la descripción de las principales etapas quirúgicas en una fundoplicatura de Nissen laparoscópica y discutir las evidencias científicas actuales que avalan los diferentes pasos en la técnica quirúrgica.

\section{Material y Método}

\section{Pacientes estudiados}

Entre enero de 1993 hasta diciembre de 2013, se han operado un total de 1.120 pacientes con reflujo gastroesofágico por vía laparoscópica en el Departamento de Cirugía del Hospital Clínico de la Universidad de Chile. De este universo, un total de 650 pacientes $(58 \%)$ fueron sometidos a una fundoplicatura de Nissen laparoscópica. El resto de los pacientes corresponde a la realización de una gastropexia posterior con calibración cardial por vía laparoscópica. El presente reporte es un estudio prospectivo observacional de casos consecutivos operados con la misma técnica, ya que desde el $1^{\text {er }}$ paciente operado se confeccionó una ficha especial en la que se traspasaban todos los datos del paciente para su posterior control alejado. De esta manera se ha acumulado una gran cantidad de información. Los pacientes sometidos a fundoplicatura de Nissen laparoscópica correspondieron a:

a. Reflujo gastroesofágico patológico: 503 pacientes.

b. Hernia hiatal tipo I-II-III y IV mayores a $5 \mathrm{~cm}$ : 115 pacientes.

c. Esófago de Barrett Corto: 32 pacientes.

Los resultados alejados de esta técnica laparoscópica en pacientes con Barrett corto ya han sido publicados 9 . Los pacientes con hernias hiatales $>5$ $\mathrm{cm}$ que en la mayoría requirieron del uso de malla serán analizados en otra publicación por su mayor complejidad. Por lo tanto, el presente estudio se referirá solamente a los 503 pacientes con reflujo gastroesofágico y/o con una hernia hiatal tipo I menor a $5 \mathrm{~cm}$.

\section{Evaluación preoperatoria}

Todos los pacientes fueron sometidos a una evaluación clínica acuciosa, a un estudio endoscópico con toma de biopsias yuxtacardiales, manometría esofágica y pHmetría de $24 \mathrm{~h}$, tal como ha sido ampliamente descrito en numerosas publicaciones previas $^{10-14}$.

\section{Técnica quirúrgica}

Para la posición de pacientes se emplea la posición de Grassi con el tórax más levantado que el abdomen mediante un cojín especialmente diseñado para este propósito. La operación tiene 6 etapas principales:

a. Colocación de trocares: se emplean 5 trocares, 2 de $5 \mathrm{~mm}$ y $3 \mathrm{de} 10 \mathrm{~mm}$. El primer trocar por la cámara se coloca $2-3 \mathrm{~cm}$ a la izquierda de la línea media supraumbilical, en la mitad entre el ombligo y el xifoides. Posteriormente un trocar de 5 $\mathrm{mm}$ subxifoídeo para separar el hígado, un trocar de $10 \mathrm{~mm}$ en línea medio clavicular izquierda, a $5-7 \mathrm{~cm}$ bajo la parrilla costal, un trocar de $10 \mathrm{~mm}$ en región costal izquierda y uno de $5 \mathrm{~mm}$ en línea medio clavicular derecha 5 a $7 \mathrm{~cm}$ por debajo de la parrilla costal derecha.

b. Disección de la unión gastroesofágica: se comienza con disección del ángulo de His hasta ver el pilar izquierdo del diafragma. Enseguida se diseca la curva menor 2-3 cm distal a la unión gastroesofágica, similar a la vagotomía supraselectiva para preservar el nervio de Latarjet, disecando la cara anterior y posterior de la unión gastroesofágica (abordaje derecho y posterior), seccionando la membrana freno-esofágica ${ }^{10,13}$. De esta manera los troncos anterior y posterior del nervio vago se desplazan hacia la derecha y hacia proximal, junto con las ramas hepática y celiaca.

c. Para poder obtener un fondo gástrico suelto, sin tensión, se seccionan 2 vasos cortos que van desde la cara posterior del fondo gástrico hasta el retroperitoneo y el primer vaso corto que va desde el estómago hacia el bazo. Esto permite obtener una fundoplicatura suelta (Floppy Nissen $)^{3}$.

d. Cierre pilares: esta fase del procedimiento quirúrgico se realiza siempre con al menos 3 puntos de seda 0 , aún cuando no esté dilatado el hiato esofágico. Hay que tener especial cuidado de visualizar y preservar el vago posterior. 
e. Finalmente se realiza la fundoplicatura total de $360^{\circ}$, de $4 \mathrm{~cm}$ de largo, sobre una bujía de 42 $F$ para evitar una estenosis o una plicatura muy suelta. El punto más proximal y el más distal se anclan en el esófago ${ }^{12}$.

f. Se colocan finalmente 1 a 2 puntos de fundofrenopexia anterior con sutura no absorbible para evitar una posterior hernia paraesofágica iatrogénica $^{13}$.

\section{Resultados}

En la Tabla 1 se muestra la cantidad total de pacientes con reflujo gastroesofágico sometidos a fundoplicatura laparoscópica desde 1993 hasta diciembre de 2013, que corresponde a un período de 21 años. La evaluación preoperatoria completa se realizó en el 100\% de los pacientes candidatos a cirugía. El estudio radiológico sólo se efectúa en el $14 \%$ de los casos. La Tabla 2 señala las principales características clínicas de estos pacientes: predominan las mujeres sobre los hombres, con una edad promedio de 45 años, con típicos síntomas de reflujo gastroesofágico y con una larga duración de síntomas. Ningún paciente tenía menos de 36 meses de sintomatología y era dependiente de inhibidores de la bomba de protones.

La Tabla 3 describe los detalles intraoperatorios. La duración promedio es una hora y media. Hubo en este grupo 2 conversiones debido a adherencias múltiples abdominales. No hubo esplenectomía ni lesión de bazo. Los vasos cortos se seccionaron en el $100 \%$ de los casos. En un paciente ocurrió una perforación del esófago distal con la introducción de la bujía, que requirió suturas del esófago y co-

Tabla 1. Fundoplicatura laparoscópica de Nissen (1993-2013)

Total casos operados $=\mathbf{5 0 3}$

\begin{tabular}{|lc|}
\hline Evaluación preoperatoria & \% \\
\hline Clínica & 100 \\
\hline Endoscopia & 100 \\
\hline Biopsias & 100 \\
Manometría & 100 \\
pH de 24 h & 100 \\
\hline Radiología & 14 \\
\hline
\end{tabular}

bertura con la fundoplicatura. La Tabla 4 muestra la evolución postoperatoria, hubo 2 pacientes quienes requirieron reoperación: Uno por un hemoperitoneo $\mathrm{y}$ en un paciente con disección de la curva menor ocurrió una necrosis localizada con perforación, que requirió reoperación y sutura. La evolución de todos los pacientes reoperados fue satisfactoria. No hubo mortalidad operatoria.

Tabla 2. Características clínicas de pacientes sometidos a fundoplicatura de Nissen $\mathbf{N}=\mathbf{5 0 3}$

\begin{tabular}{|l|l|}
\hline Hombres & $32 \%$ \\
\hline Mujeres & $68 \%$ \\
\hline Edad (años) & $45 \pm 14$ \\
\hline Pirosis & $92 \%$ \\
\hline Regurgitación & $76 \%$ \\
\hline Disfagia & $15 \%$ \\
\hline Duración síntomas (meses) & $72 \pm 23$ \\
\hline
\end{tabular}

Tabla 3. Detalles operatorios de la fundoplicatura de Nissen laparoscópica $\mathbf{N}=\mathbf{5 0 3}$

\begin{tabular}{|ll|}
\hline Tiempo operatorio & $85 \pm 25 \mathrm{~min}$ \\
\hline Conversión a laparotomía & $2(0,4 \%)$ \\
\hline Esplenectomía & 0 \\
\hline Sección vasos cortos & $100 \%$ \\
\hline Perforación del esófago distal por bujía & $1(0,2 \%)$ \\
\hline
\end{tabular}

Tabla 4. Evolución postoperatoria $\mathbf{N}=\mathbf{5 0 3}$

\begin{tabular}{|ll|}
\hline Hemoperitoneo reoperado & $1(0,2 \%)$ \\
\hline Necrosis curva menor reoperada & $1(0,2 \%)$ \\
\hline Días estada hospitalaria & $2,8 \pm 0,5$ \\
\hline Mortalidad & 0 \\
\hline
\end{tabular}


Tabla 5. Resultados nacionales de la fundoplicatura laparoscópica

\begin{tabular}{|c|c|c|c|c|c|c|}
\hline Autor & Tipo cirugía & $\mathbf{n}$ & Morbilidad & Mortalidad & Conversión & Tipo paciente \\
\hline Larraín (1995) & $\mathrm{CC}+\mathrm{GP}$ & 42 & $5 \%$ & 0 & $3(7,1 \%)$ & RGE \\
\hline Awad (1995) & Nissen & 66 & 0 & 0 & $2(3 \%)$ & RGE \\
\hline Csendes (1996) & Nissen & 32 & 0 & 0 & 0 & RGE \\
\hline Braghetto (1998) & $\mathrm{CC}+\mathrm{GP}$ & 15 & $7 \%$ & 0 & 0 & RGE \\
\hline Contreras (1998) & Nissen-Rosetti & 50 & $12 \%$ & 0 & $4(8 \%)$ & RGE \\
\hline Hernández (2000) & Nissen & 53 & $6 \%$ & 0 & $2(3,8 \%)$ & RGE \\
\hline Csendes (2001) & Nissen & 108 & $1 \%$ & 0 & 0 & $\begin{array}{l}\mathrm{RGE}=99 \\
\mathrm{BC}=9\end{array}$ \\
\hline Manterola (2001) & Nissen-Rosetti & 24 & $8,3 \%$ & 0 & 0 & RGE \\
\hline Csendes (2005) & Nissen & 225 & $0,4 \%$ & 0 & $3(1,3 \%)$ & $\begin{array}{l}\mathrm{RGE}=198 \\
\mathrm{HH}=27\end{array}$ \\
\hline Braghetto (2005) & $\mathrm{CC}+\mathrm{GP}$ & 108 & $1 \%$ & 0 & 0 & $\begin{array}{l}\mathrm{RGE}=83 \\
\mathrm{BC}=12 \\
\mathrm{HH}=13\end{array}$ \\
\hline Braghetto (2013) & $\mathrm{CC}+\mathrm{GP}$ & 343 & $4,7 \%$ & 0 & 0 & $\begin{array}{l}\mathrm{RGE}=193 \\
\mathrm{BC}=25 \\
\mathrm{HH}=125\end{array}$ \\
\hline Csendes (2015) & Nissen & 530 & $0,4 \%$ & 0 & $2(0,4 \%)$ & $\mathrm{RGE}=503$ \\
\hline
\end{tabular}

$\mathrm{CC}+\mathrm{GP}=$ Calibración cardial + gastropexia posterior. $\mathrm{RGE}=$ Reflujo gastroesofágico. $\mathrm{BC}=$ Barrett corto. $\mathrm{HH}=\mathrm{Hernia}$ hiatal.

\section{Discusión}

Los resultados del presente estudio sugieren que la fundoplicatura de Nissen por vía laparoscópica es una operación muy bien tolerada y con muy escasos efectos adversos en el postoperatorio inmediato, que fue el objetivo del presente estudio. Las opciones de tratamiento para la enfermedad por reflujo gastroesofágico ${ }^{5}$ se basan en terapia médica (inhibidores de la bomba de protones o bloqueadores $\mathrm{H}_{2}$ ) o quirúrgica (fundoplicatura). Las indicaciones de la cirugía ${ }^{9,14}$, incluyen a pacientes bajo 60 años, que estando asintomáticos con terapia médica, recurren muy precozmente al suspenderlos, siempre que se demuestre un reflujo ácido patológico y un esfínter incompetente. La controversia entre terapia médica y quirúrgica ha sido resuelto por la evaluación de 7 estudios prospectivos y aleatorizados, que han demostrado que la cirugía es una alternativa al tratamiento médico para pacientes con un control sintomático o con alivio sintomático parcial con una evidencia nivel $1^{5,15}$.

La siguiente pregunta consiste en conocer si la fundoplicatura laparoscópica es superior o a lo menos similar en resultados comparada con el abordaje laparotómico. Esta inquietud también se ha demostrado en 10 estudios prospectivos y aleatorizados, concluyendo que la vía laparoscópica es superior al abordaje tradicional en el corto plazo, con todas las ventajas que implica la cirugía mínimamente invasiva ${ }^{16}$.

Nuestro estudio confirma que este procedimiento está asociado a una morbilidad muy baja, una permanencia hospitalaria muy corta y a un excelente pronóstico. La fundoplicatura laparoscópica total de $360^{\circ}$ está considerada hoy el procedimiento de elección debido a que aumenta la presión de reposo del esfínter esofágico inferior, aumenta el largo del esfínter, disminuye las relajaciones transitorias y mejora la calidad de la peristalsis esofágica ${ }^{17}$. Las complicaciones más graves en el postoperatorio inmediato son la perforación esofágica o gástrica $o$ una lesión al bazo

La perforación del esófago distal ocurre generalmente durante la introducción de la bujía de calibración. En este momento hay que tener cuidado en 2 detalles: a) la crura diafragmática no debe estar muy apretada, sino sólo ajustada alrededor del esófago, permitiendo deslizar una pinza fácilmente entre el esófago y la crura. El cierre generoso de la crura crea un ángulo agudo entre el esófago y el estómago, facilitando una perforación. b) el otro detalle es la tracción hacia distal de la unión gastroesofágica y la apertura del drenaje de Penrose o de la sonda Nelaton que es la que tracciona la unión gastroesofágica, para permitir el paso fácil de la bujía. 
La perforación gástrica puede ocurrir por la tracción forzada del fondo gástrico por una pinza o por quemadura del fondo durante la sección de los vasos cortos.

Si ocurre una lesión del bazo, hay que evaluarla con prudencia para evitar al máximo la esplenectomía. Con alguna frecuencia ocurre un pequeño desgarro del bazo que se controla con electrocoagulación y materiales coagulantes ${ }^{18}$. En esta serie no ha ocurrido una esplenectomía.

En la Tabla 5 se resume toda la experiencia chilena publicada sobre los resultados inmediatos de la fundoplicatura de Nissen por laparoscopia, ya que los resultados a largo plazo ya han sido comunicados $^{8}$. Hay 7 autores que han reportado sus resultados en 12 publicaciones ${ }^{9,12,18,19-26}$. Se han empleado básicamente 3 técnicas: la calibración cardial con gastropexia posterior ${ }^{27}$, la fundoplicatura clásica de Nissen ya descrita y la técnica de Nissen-Rosetti ${ }^{28}$, técnica que no contempla la sección de los vasos cortos y utiliza la cara anterior del fondo gástrico para efectuar la fundoplicatura. Se puede apreciar que la mortalidad operatoria en todos los trabajos han sido nula, que la conversión a cirugía laparotómica es muy baja en la medida que se adquiere experiencia con esta técnica y que las complicaciones postoperatorias son muy bajas, lo que confirma que esta técnica laparoscópica es muy segura, con resultados incluso superiores a la vía laparotómica.

Las complicaciones reportadas por los autores corresponden a ectasia gástrica, hemorragia digestiva, colección subfrénica, hemoperitoneo, hemotórax izquierdo, afagia total, necrosis curva menor, reoperaciones, etc., pero que ninguno causó mortalidad. Las reoperaciones precoces ocurren en pacientes con hemoperitoneo, afagia aguda y atonía gástrica.

En la literatura internacional se describe la conversión a cirugía abierta entre 0 a $24 \%$, siendo la conversión en centros de alto volumen menor a $4 \%{ }^{29-32}$. Las complicaciones reportadas son enteramente similares a los descritos por autores nacionales. La perforación esofágica y gástrica ocurren entre 0 a $4 \%{ }^{30-44}$. Neumotórax ha ocurrido muy raramente en Chile, pero su incidencia se reporta entre 0 a $1,5 \% \%^{31,32,35-37,39,42,45-47}$. La necesidad de una re-intervención en esta circunstancia es muy rara ya que la injuria sólo compromete a la pleura pero sin daño pulmonar.

Hay 2 pasos quirúrgicos que requirieron una atención especial:

1. División de los vasos cortos: hay 5 estudios prospectivos y aleatorios que han evaluado el impacto de la sección de estos vasos cortos sobre el resultado de la cirugía antireflujo sobre el resultado de la cirugía antireflujo ${ }^{48-52}$. Las conclusiones sugeridas son que los resultados son similares con o sin sección de vasos cortos. Sin embargo, la revisión actual $^{53}$ de todos los estudios y de la opinión de expertos cirujanos de USA recomienda la decisión rutinaria de los vasos cortos, que es y ha sido nuestro proceder en todos los pacientes.

2. Cierre o no del hiatus esofágico, en casos con reflujo gastroesofágico sin hernia hiatal. No hay estudios prospectivos y aleatorios sobre este punto. Hay reportes que señalan que es beneficioso su cierre $^{54-56}$, pero para otros no hay diferencia ${ }^{51,57-59}$. Nuestra postura ha sido cerrar el hiato siempre, respetando el vago posterior, ya que durante la cirugía hay una importante disección de la región de la unión gastroesofágica, lo que facilita la aparición de una hernia posterior a la cirugía, si no se cierra el hiato.

La operación de Nissen-Rossetti no ha convencido a los cirujanos como superior al Nissen clásico. De hecho, las complicaciones intraoperatorias son mayores $(3,1 \%)$, la conversión es de $6,6 \%$ y las complicaciones postoperatorias cercanas al $4 \%$, cifras superiores a los observados con la fundoplicatura convencional ${ }^{61}$.

Por otra parte, la comparación de una plicatura de $360^{\circ}$ (Nissen) versus $270^{\circ}$ (Toupet) ha mostrado resultados similares, pero con más altas tasas de complicaciones postoperatorias en la última técni$\mathrm{ca}^{62}$. Finalmente la cirugía robótica no ha mostrado ventaja alguna sobre la fundoplicatura convencional, siendo de mayor costo ${ }^{63}$.

En conclusión, la fundoplicatura de Nissen laparoscópica es una operación segura, de muy baja morbimortalidad, en pacientes con síntomas de reflujo gastroesofágico crónico, con o sin una hernia hiatal.

\section{Referencias}

1. DeMeester TR, Bonavina L, Albertucci M. Nissen fundoplication for gastroesophageal reflux disease. Ann Surg. 1986;204:9-20.

2. Dent J, Serag HB, Wallander MA, Johansson S. Epidemiology of gastroesophageal reflux disease: A systematic review. Gut 2005;54:710-7.

3. Davis RE, Awad ZT, Felipi CJ. Technical factors in the creation of a "floppy" Nissen fundoplication. Am J Surg. 2004;187:724-7.

4. Dallemagnee B, Weerts J, Markiewicz S. Clinical results of laparoscopic fundoplication ten years after surgery. Surg Endosc. 2006;20:159-65.

5. Wileman S, McCann S, Grant AM, Krukowski ZH, Bruce J. Medical versus surgical management for gastroesophageal reflux disease in adults. Cochrane Database System 2010;3:CD003243.

5. Csendes A, Álvarez E, Burdiles P, Braghetto I. Magnitud del reflujo gastroesofágico ácido cuantificado por medicación de $\mathrm{pH}$ de 24 h según grado de esofagitis. Rev Med Chile 1994;122:59-67. 
6. Neufeld M, Graham A. Levels of evidence available for techniques in antireflux surgery. Dis Esoph. 2007;20:161-7.

7. Csendes A, Burdiles P, Korn O, Braghetto I, Huertas C, Rojas J. Late results of a randomized clinical trial comparing total fundoplication versus calibration of the cardia with posterior gastropexy. Brit J Surg. 2000;87:289-97.

8. Csendes A. Resultados alejados (10 años) de la fundoplicatura de Nissen en pacientes con reflujo gastroesofágico sin esófago de Barrett. Rev Chil Cir. 2012;64:4836.

9. Csendes A, Braghetto I, Burdiles P, Smok G, Henríquez A, Burgos AM. Late results of the surgical treatment of 125 patients with short segment Barrett's esophagus. Arch Surg. 2009;144:921-7.

10. Csendes A, Burdiles P, Díaz JC, Rojas J. Resultados de la cirugía antirreflujo por videolaparoscópica en 108 pacientes. Rev Chil Cir. 2001;53:487-94.

11. Csendes A, Henríquez A, Quesada S. Estudio continuo de $\mathrm{pH}$ intraesofágico por $24 \mathrm{~h}$. Rev Chil Cir. 1993;45:94-6.

12. Csendes A, Álvarez E, Burdiles P, Braghetto I. Magnitud del reflujo gastroesofágico cuantificado por medición de $\mathrm{pH}$ de 24 h según grado de esofagitis. Rev Med Chile 1994;122:59-67.

13. Csendes A, Burdiles P, Korn O. Laparoscopic Nissen fundoplication: the "right posterior" approach. J Gastrointest Surg. 2005;9:985-91.

14. Csendes A, Burdiles P, Álvarez F. Manometric features of mechanically defective lower esophageal sphincter in controls in patients with different degrees of gastroesophagal reflux disease. Dis Esoph. 1996;9:290-4.

15. Campos MR, Peters JM, DeMeester R. Multivariate analysis of factors predicting outcome after laparoscopic Nissen fundoplication. J Gastrointest Surg. 1999;3:292300.

16. Csendes A. ¿Cuándo se indicó y cuáles son los resultados a largo plazo de la cirugía en RGE sin hernia hiatal verdadera? Acta Latinom Gastroent. 2015 (en prensa).

17. Salvinen PTP, Hiekkanen HI, Rantala AP, Ovaska JT. Comparison of long term outcome of laparoscopic and conventional Nissen fundoplication. A prospective randomized study with an 11 year follow up. Ann Surg 2007;246:201-6.

18. Herbella FA, Tedesco P, Niponnick F, Patti HG. Effect of partial and total laparoscopic fundoplication on esophageal body motility. Surg Endosc. 2007;21:285-9.

19. Braghetto I, Korn O, Cardemil G, Valladares H, Masia G, Mandiola C. Resultados del tratamiento quirúrgico de la patología esofágica benigna con cirugía mínimamente invasiva. Experiencia en 483 pacientes. Rev Chil Cir. 2013;65:128-38.

20. Larraín A, Carvajal C, Valenzuela H, Ceresa S, Vargas P. Calibración cardial por laparoscopia. Evaluación clínica. Rev Chil Cir. 1995;47:115-7.
21. Awad W, Loehnert R. Vagotomía supraselectiva laparoscópica y corrección del reflujo gastroesofágico. Rev Chil Cir. 1995;47:537-45.

22. Csendes A, Burdiles P, Díaz JC, Maluenda F, Korn O. Evaluación subjetiva y objetiva de los resultados de la cirugía antirreflujo por vídeo laparoscópica en pacientes con reflujo gastroesofágico. Rev Med Chile 1996; 124; 1077-85.

23. Braghetto I, Korn O, Debandi A, De La Cuadra R. Calibración cardial y vagotomía supraselectiva con cinco trocares en reflujo gastroesofágico patológico. Rev Chil Cir. 1998;50:148-55.

24. Contreras JE. Fundoplicatura de Nissen laparoscópica en el tratamiento del reflujo gastroesofágico patológico. Rev Chil Cir. 1998;50:156-74.

25. Hernández F, Leiva PL, Umaña M, Collis JP, Matus FC, Cerda SR. Fundoplicatura de Nissen laparoscópica vs convencional. Rev Chil Cir. 2000;52:487-94.

26. Manterola C, Gutiérrez R, Soto A, Cuadra A. Modificación de variables clínica y de laboratorio en pacientes sometidos a cirugía antirreflujo. Rev Chil Cir. 2001;53:287-92.

27. Braghetto I, Korn O, Debandi A, Burdiles P, Valladares H. Laparoscopic cardial calibration and gastropexy for treatment of patients with reflux esophagitis. World J Surg. 2005;29:636-44.

28. Larrain A. Technical consideration in posterior gastropexy. Surg Gynec Obs. 1971;132:299-300

29. Rosetti M, Hell K. Fundoplication for the treatment of gastroesophageal reflux in hiatus hernia. World J Surg. 1977;1:439-44.

30. Anvari M, Allen C, Marshall J, Armstrong D, Goeree $\mathrm{R}$, Ungar $\mathrm{W}$, et al. A randomized controlled trial of laparoscopic Nissen fundoplication versus proton pump inhibitors for treatment of patients with chronic gastroesophageal reflux disease. One year follow up. Surg Innov. 2006;13:238-49.

31. Mahon D, Rhodes M, Decadt B, Hindmarsh A, Lowndes R, Beckingham I, et al. Randomized clinical trial of laparoscopic Nissen fundoplication compared with proton-pump inhibitors for treatment of chronic gastrooesophageal reflux. Brit J Surg. 2005;92:695-9.

32. Mehta S, Bennett J, Mahon D, Rhodes M. Prospective trial of laparoscopic Nissen fundoplication versus proton pump inhibitor therapy for gastroesophageal reflux disease: Seven year follow up. J Gastrointest Surg. 2006;10:1312-6.

33. Anvari M, Allen C, Borm A. Laparoscopic Nissen fundoplication is a satisfactory alternative to long term omeprazole therapy. Brit J Surg. 1995;82:938-42.

34. Zaninotto G, Portale G, Costantini M, Rizzetto C, Guirroli, Ceolin M, et al. Long term results (6-10 years) of laparoscopic fundoplication. J. Gastrointest Surg. 2007; 11: 1138-45.

35. Pessaux P, Arnaud JP, Delattre J, Meyer C, Bauliewx J, Mosnier H. Laparoscopic antireflux surgery: five 
year results and beyond in 1340 patients. Arch Surg. 2005; 140:946-951.

36. Anvari M, Allen C. Five years comprehensive outcomes evaluation in 181 patients after laparoscopic Nissen fundoplication. J Am Coll. 2003;196:51-7.

37. Gad Ed-Hak N, Abo Zied M, Aboelenen A, Fouad A, Abd Alla T, El-Shoubary M, et al. Short gastric vessels division in laparoscopic Nissen fundoplication. Hepatogastroenterology 2005;52:1742-7.

38. Gotley DC, Smithers BM, Rhodes M, Menzies B, Branicki FJ, Nathanson L. Laparoscopic Nissen fundoplication 200 consecutive cases. Gut 1996;38:487-91.

39. Victorzon M, Tolonen P. Symptomatic outcome of laparoscopic fundoplication, using a minimal dissection technique. Scand J Surg. 2003;92:138-43.

40. Kala Z, Dolina J, Kysela P, Hermanova M, Prochazka $\mathrm{V}$, Kroupa R, et al. Intraoperative manometry of the lower esophageal sphincter pressure during laparoscopic antireflux surgery with a mechanical calibration-early results. Hepatogastroenterology 2006;53:710-4.

41. Morino M, Pellegrino L, Giaccone C, Garrone C, Rebecchi F. Randomized clinical trial of robot assisted versus laparoscopic Nissen fundoplication. Bri J Surg. 2006;93:553-8.

42. Anvari M, Bamehriz F. Outcome of laparoscopic Nissen fundoplication in patients with body mass index $>$ or $=$ 35. Surg Endosc. 2006;20:230-4.

43. Cowgill SM, Gillman R, Kraemer E, Al-Saadi S, Villadolid D, Rosemurgy A. Ten year follow up after laparoscopic Nissen fundoplication for gastroesophageal reflux disease. Am Surg. 2007;73:748-52.

44. Desai KM, Frisella MM, Soper NJ. Clinical outcomes after laparoscopic antireflux surgery in patients with and without preoperative endoscopic esophagitis. J Gastrointest Surg. 2003;7:44-51.

45. Fernando HC, Schauer PR, Buenaventura PO, Christie NA, Close JM, Luketich JD. Outcomes of minimally invasive antireflux operations in the elderly: a comparative review. JSLS 2003;7:311-5.

46. Tedesco P, Lobo E, Fisichella PM, Way LW, Patti MG. Laparoscopic fundoplication in elderly patients with gastroesophageal reflux disease. Arch Surg. 2006;141:289-92.

47. Vidal O, Lacy AM, Pera M, Valentini M, Bollo J, Lacima $\mathrm{G}$, et al. Long term control of gastroesophageal reflux disease symptoms after laparoscopic Nissen-Rosetti fundoplication. J Gastrointest Surg. 2006;10:863-9.

48. Granderath FA, Granderath UM, Pointner R. Laparoscopic revisional fundoplication with circular hiatal mesh prosthesis: the long term results. World J Surg. 2008;32:999-1007.

49. Yang H, Watson D, Lally C, Devitt P, Game P, Jamieson G. Randomized trial of division versus nondivision of the short gastric vessels during laparoscopic Nissen fundoplication: 10-year outcomes. Ann Surg. 2008;247:38-42.

50. Engstrom C, Blomqvist A, Dalenback J, Lonroth H,
Ruth M, Lundell L. Mechanical consequences of short gastric vessel division at the time of laparoscopic total fundoplication. J Gastrointest Surg. 2004;8:442-7.

51. O`Boyle CJ, Watson DI, Jamieson GG, Myers JC, Game PA, Devitt PG. Division of short gastric vessels at laparoscopic Nissen fundoplication: a prospective double blind randomized trial with 5 year follow up. Ann Sur. 2002;235:165-70.

52. Blomqvist A, Dalenback J, Hagedorn C, Lonroth H, Hyltander A, Lundell L. Impact of complete gastric fundus mobilization on outcome after laparoscopic total fundoplication. J Gastrointest Surg. 2000;4:493-500.

53. Chrysos E, Tzortzinis A, Tsiaoussis J, Athanasakis H, Vasssilakis J, Xynos E. Prospective randomized trial comparing Nissen to Nissen-Rossetti technique for laparoscopic fundoplication. Am J Surg. 2001;182:215-21.

54. Neufeld M, Graham A. Levels of evidence available for techniques in antireflux surgery. Dis Esophagus 2007;20:161-7.

55. Donahue PE, Larson GM, Stewardson RH, Bombeck CT. Floppy Nissen fundoplication. Rev Surg. 1977;34:223-4.

56. DeMeester TR, Bonavina L, Albertucci M. Nissen fundoplication for gastroesophageal reflux disease. Evaluation of primary repair in 100 consecutive patients. Ann Surg. 1986;204:9-20.

57. Wu JS, Dunnegan DL, Luttmann DR, Soper NJ. The influence of surgical technique on clinical outcome of laparoscopic Nissen fundoplication. Surg Endosc. 1996;10:1164-9.

58. Watson DI, Pike GK, Baigrie RJ, Mathew G, Devitt PG, Britten-Jones R, et al. Prospective double blind randomized trial of laparoscopic Nissen fundoplication with division and without division of short gastric vessels. Ann Surg. 1997;226:642-52.

59. Anvari M, Allen C. Laparoscopic Nissen fundoplication: two year comprehensive follow up of a technique of minimal paraesophageal dissection. Ann Surg. 1998;227:25-32.

60. Watson DI, Jamieson GG, Devitt PG, Kennedy JA, Ellis $\mathrm{T}$, Ackroyd R, et al. A prospective randomized trial of laparoscopic Nissen fundopliction with anterior vs posterior hiatal repair. Arch Surg. 2001;136:745-51.

61. Moral MG, Reoyo PJ, León MR, Palomo LA, Rodríguez SS, Seco GJ. Fundoplicatura de Nissen laparoscópica. Resultados y factores pronóstico. Rev Gastroent México 2012;77:15-25.

62. Tian Z, Wang B, Shan CX, Zhang W, Jiang DZ, Qiu M. A meta-analysis of randomized controlled trials to compare long term outcomes of Nissen and Toupet fundoplication for gastroesophageal reflux disease. PloS One 2015;10:1-15.

63. Yao G, Lin K, Fan L. Robotic Nissen fundoplication for gastroesophageal reflux disease: a meta analysis of prospective randomized controlled trials. Surg Today 2014;44:1415-21. 